\title{
What Is a Muon Anyways: Meson or Lepton!?
}

\author{
Lucian M. Ionescu \\ Department of Mathematics, Illinois State University, IL, USA \\ Email:1miones@ilstu.edu
}

How to cite this paper: Ionescu, L.M. (2020) What Is a Muon Anyways: Meson or Lepton!? Journal of High Energy Physics, Gravitation and Cosmology, 6, 244-250. https://doi.org/10.4236/jhepgc.2020.62018

Received: March 7, 2020

Accepted: March 29, 2020

Published: April 2, 2020

Copyright (c) 2020 by author(s) and Scientific Research Publishing Inc. This work is licensed under the Creative Commons Attribution International License (CC BY 4.0).

http://creativecommons.org/licenses/by/4.0/

\begin{abstract}
Understanding the role of muons in Particle Physics is an important step understanding generations and the origin of mass as an expression of "internal structure". A possible connection between muonic atoms and cycloatoms is used as a pretext to speculate on the above core issue of the Standard Model.
\end{abstract}

\section{Keywords}

Standard Model, Cycloatoms, Elementary Particles, Muons

\section{Introduction}

The Standard Model is an impressive mody of Physics knowledge, deeply rooted in experiment with its impressive particle accelerators: no doubt about it! ... except for some natural questions still lurking: "Who ordered that?" (about the discovery of the muon); and "What is mass, really?", beyond the Higgs mechanism within a Lagrangean formulation of Gauge FIELD Theory (Do we still believe in "fields"!?), while the evidence that mass is quantized, abounds (e.g. [1] [2]). But this is another story (to come [3]), so let's ponder upon a basic question in particle physics ...

What is a muon? It comes from a meson (and was originally classified as such [4]), but declares itself, deep-down, to be a lepton at heart ${ }^{1}$ :

$$
\pi \rightarrow \mu+\bar{v}_{\mu}, \quad \mu+\bar{v}_{\mu} \rightarrow e^{-}+\bar{v}_{e},
$$

as shown by the above "particle reaction" diagrams ${ }^{2}$.

Of course, historically speaking (e.g. [4] [6]), the lack of interaction with matter of the muon, "fueled" to the discovery of the pion, in order to substantiate Yukawa's theory of nuclear force, which now is derived from a basic, yet purely theoretical QCD, but which "works" well. Nevertheless, until the nature of quark ${ }^{1}$ The diagrams are not typical, but modulo cross-symmetry, they are allowed; see Wiki [5] [6]. ${ }^{2}$ Feynman diagrams without propagators and coupling constants: like in chemical reactions, just accounting for the bare ingredients. 
flavor is explained in a satisfactory way, including mass spectrum and number of generations, alternatives should be explored ${ }^{3,4}$.

Note that the decay of the muon involves the weak interaction "bosons" $W^{-}$, as shown in quark line diagrams, and not the photon $\gamma$, as in a simple EM transition between atomic orbitals (SM).

What's the "difference"?5 Ditto for the pion decay, not a strong interaction, as in the many strange mesons production processes (bumping quarks one generation up), the weak interaction bosons are responsible for the quark flavor transition.

Remark 1.1. It is too early to ask, or claim anything, but let us keep in mind the short exact sequence from the footnote: $U(1) \rightarrow U(2) \rightarrow S U(2)$, in connection with the Electro-Weak Theory. Still, it's as if $W^{-}$is the "electron", but with a mass reflecting an internal structure, modeled algebraically as a state in an irreducible representation of the larger group $S U(2)$, and the antineutrino corresponds to the section splitting the sequence ...

With these thought in author's mind, reading about cycloatoms [7], which are high energy relativistic effects where the electronic orbitals are molded into new, topologically non-trivial structures, raised another thought: is the laser pumping enough energy to materialize, for example, the toroidal structure of the electron's orbital, in the confining presence of strong magnetic fileds, comparable may be with those of cyclotrons, and yield none other then a muonic atom!?

So, let's review, as non-expert cycloatom and see what comes out of this ... This is the "throwing of the stone"; the burden of proof, or rather hope, is on the expert reader side ...

\section{Cycloatoms}

Computer simulations of classical and quantum mechanical time evolution of the atom in strong magnetic field, excited through a powerful laser [7], have shown resonant relativistic bound states for the electron, with topologies different from that of the usual atomic orbitals, as for instance the $(n, l)$-subshell solutions of Schrodinger equation, e.g. figure eight or propelar-like distributions [9] [10], which were collectively called cycloatoms.

Exciting an electron coherently via a laser may be compared with a pion decay, or beta decay, or even better, with the decay of a muonic atom [6].

The initial spiraling of the atoms (see the movies of cycloatoms in loc. cit.) is the transient phase, before reaching a standing wave of higher mass, as a resonant bound state (see movies available loc. cit.).

The advantage of using strong magnetic fields is to allow for relatively low ${ }^{3}$ There are currently communities of astrophysicists still calling it "muon meson".

${ }^{4}$ There are also other reasons why the $\mu$ meson is different from all other mesons, consistently modeled as a quark-antiquark pair [6], p.3, e.g. the number of neutrinos involved in their decay; but this is SM dependent ...

${ }^{5} \mathrm{It}$ 's in fact a quotient, i.e. short exact sequence of Gauge Groups: braking the symmetry $U(1) \rightarrow U(2) \rightarrow S U(2)$, which splits etc.: Glasghow-Weinberg-Salam Electroweak Theory.

${ }^{6}$ Perhaps the question is "crazy" enough, to have some truth to it! [8]. 
energy lasers to "pump" coherently energy, obtaining the same results as high-end lasers [11]. The emited phonos being are much more energetic then the infrared ones in the laser used, indicates a gain of 200 times, in this "micro-cyclotron" accelerator". The harmonics obtained are 5000 times the normal size of the atom, indicating a "trapping" of the electron by the magnetic field reminiscent of a "black hole".

Remark 2.1. The EM gauge vector potential A determines the geometric connection, and what the "geodesics" are, if we place ourselves in a Kaluza-Klein context, following Einstein's pioneering work with his General Relativity.

This magnetic "curving of local-space" alters therefore the bound states, allowing for much larger principal quantum numbers $n$ shells. What is unexpected though, is the topology of such an "orbital", a genus $g=1$ (not simply connected $)^{7}$

Remark 2.2. It would be interesting to test whether even stronger fields may compress the orbital to behave like a muonic atom; associating the experiment with a particle detector for a non-EM decay $\mu^{-} \rightarrow e^{-}+\bar{n} u_{e}+v_{\mu}$, is an afterthought ...

Note that the computations use purely classical relativistic mechanics, corresponding perhaps to the initial Bohr-Sommerfeld model of a moving electron, together with a lattice model approach [11]. Experimental confirmations, even indirect, are perhaps needed to validate they are still conform to a full Schrodinger equation approach, or better, with "reality".

\section{Pions and Muons}

The dominant decay mode of the pion, relating it with the muon:

$$
\pi^{-} \rightarrow \mu^{-}+\bar{v}_{\mu} .
$$

The current model (SM) resolves this process via $W$ massive boson, responsible for "carrying" the mass and charge, in a $u \rightarrow d$ quark isospin transition (corresponding $\pi^{+}$shown below): (Figure 1 ).

Remark 3.1. It would be interesting to study its the spectrum (energy or mass), and see if it can be characterized simply by an angular moment quantum number, or there is a "new" component, a multiple of the muon mass $\mu \approx 105 \mathrm{MeV} / c^{2}$, which is one of the quantum units of mass $m B=70 \mathrm{MeV}$ [2] [12].

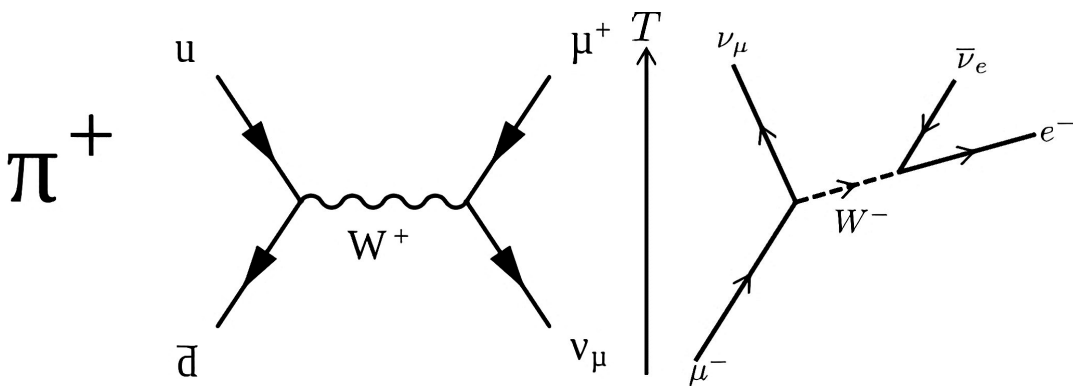

Figure 1. Decays of pion (left) and muon (right).

${ }^{7}$ Although $d_{z^{2}}$-subshells have such toroidal regions. 
Indeed, the muon mass seams to correspond to the minimal energy needed to achieve a new level of internal structure, which then is repeated in various multiples, forming the known mesons and baryons. We will develop this idea elsewhere [13].

The other decay modes of the pion, the electronic mode of decay of $\mu^{-} \rightarrow e^{-}+\bar{v}_{e}$, and the muonic beta decay $\mu^{-} \rightarrow \mu^{0}+e^{-}+\bar{v}_{e}$, are significantly suppressed on mass and helicity reasons (Branching ratios of $10^{-4}$ and $10^{-8}$ ). Yet, when comparing cycloatoms and muonic atoms, there is a certain "huntch" that there are common points.

\section{Particle-Wave Duality and Quantum Computing Networks}

The SM, built on Gauge Theory foundations around the 70s, standing strong on experimental grounds, and well correlated with the modern infrastructure, faces the birth of a new promissing paradigm: Quantum Computing, in the "new age" of Information (Classical and Quantum). It is worth pointing briefly the limitations of "believing" that leptons are "partiples", in order to "nurcher" IT (from $\left.\mathrm{Bit}^{8}\right)$, rather then neglect its future ${ }^{9}$.

Often the muon is considered simply as a "heavy electron"; then who needs it!? (paraphrasing I. I. Rabi: “Who ordered that?"). But then it must share electron's properties: atomic orbitals are "clouds", it is subject to interference as in Aharonov-Bohm experiment etc.

At this point, from HEP "particles" viewpoint, we should challenge that "electrons are pointwise"; no internal structure, yes, when performing strong measurements, like smashing things in particle accelerators, but with a lot of topological structure when arranging a coherent setup, e.g. in laser experiments. To see the huge conceptual difference between "strong measurements" and "weak measurements", as for example those in Quantum Optics, see (start with) [14], and ponder on delayed choice experiments too, besides the "old" Aharonov-Bohm experiment ${ }^{10}$.

Briefly, a holistic approach is needed (see also [15] [16]), where quantum information is processed by an I/O-Circuit, and where "time" can be defined for the classical computation "controlling" it, but not locally (anti-particles are just feedback loops).

Regarding the decay process $\pi^{-} \mapsto \mu^{-} \mapsto e^{-}$(main stages), the following "picture" (Figure 2), showing a $\Delta^{++}$production, together with a pion $\pi^{-}$, followed by the "decay" (transformation) of the pion, and then of the corresponding muon ("outputting" the negative charge), attempts to save 1000 words (to be presented elsewhere), and convey a sample of a "fat/stringy Quark Line Diagram", an analog to the well established concept of "fat" Feynman graphs ${ }^{11}$ : 


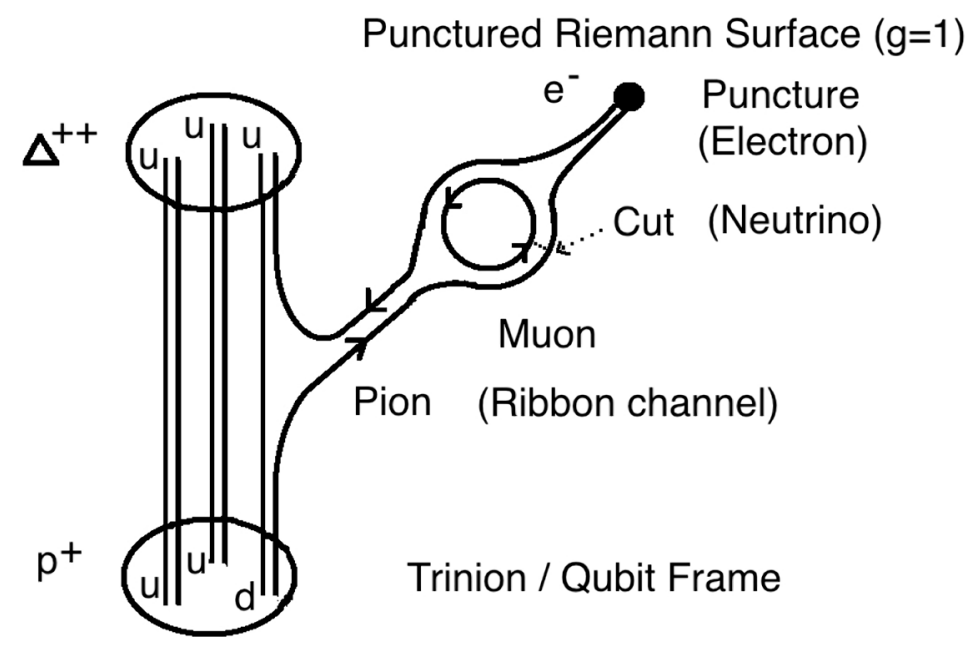

Figure 2. "Fat"' quark lines diagram of pion and muon decay.

The main point is that baryons (the above billiard-ball looking blobs representing $p^{+}$and $\Delta^{++}$), are nodes of the network (modeled as Qubit Frames [13]), mesons are quantum channels, open ribbons if joining different baryons, or closed if decaying, like solar flaires (and e.g. modeled within the framework of Turaev Graphical Calculus on ribbon categories), while electrons, muons, taons are topologically closed structures, e.g. the 2-punctured torus alluded to earlier in a double slit Aharonov-Bohm experiment, or the 1-punctured torus "glued" via a mesonic channel to the trinion representing the proton as a qubit frame, in the above picture, a rendering of what the muon seems to be (iconic level).

\subsection{What Is a Neutrino, Then?}

The "particle solution" of the beta decay, the neutrino, may be "upgraded" to a String Theory solution:

1) the continuous spectrum of energy, conflicting to a tree-level 3-body collison, and leading to Pauli's solution "there is an unseen 4th particle" (neutrino), should be re-evaluated once Feynman graphs were invented and loop corrections are ubiquitous in HEP: the loop momentum $\vec{A}$ plays the role of the unseen carrier;

2) The "multiple paths solution, in the context of an Aharonov-Bohm like experiment, involves a "collapse of the wave function", which corresponds to a cut; how to model mathematically, corresponding to the well established theory of neutrinos, remains to be seen. At this point, the mass and oscillation problems seem to have new opportunities to be explained.

Charge is a period (e.g. Gauss' Theorem), as well as amplitudes in CFT (e.g. Veneziano amplitudes).

\subsection{On the Measuring Problem in Quantum Physics}

Nevertheless measuring such an event using for instance detectors as macro-objects not quantum synchronized with the Quantum Circuit, is like poking 
a chip with a voltmeter, or a soap baloon with a needle: the pop-release of energy-matter is localized, as if the object (unseen otherwise in the case of "elementary particles") is pointwise ${ }^{12}$.

In support of the global network modeling approach, vs. moving particles in space-time, we will also mention the Special Relativity "forbidden" slow-down of light to speeds of hundred feet per second, in a cross-fire of lasers [18]. In the author's re-interpretation of quantum physics, the coherence allows a standing wave be formed, and the photon quanta (bosons), as individual pulses carrying classical information, to be slowed-down, and perhaps manipulated like a yo-yo on this unseen fermionic network, with non-trivial homology (charges as periods). "Fermions" are thus distributed as "clouds" in an orbital or Aharonov-Bohm experiment, not being "moving particles" etc.

As mentioned elsewhere [16], this natural way to unify fermions and bosons, which are aspects historically studied and modeled as separate ${ }^{13}$.

\section{Conclusions and Further Developments}

Topological aspects (structure) may turn out to be relevant in particle physics as well, in the spirit of Feynman Path Integrals and Diagrams, which are in fact the source of coherence, constructive or destructive, and leading to bound states and resonance.

More specifically, the toroidal structure of a muon as a source of its mass is compared with cycloatoms. The former is obtained in huge particle accelerators, while the later, in experimental setups consisting in a laser feeding energy into an atom subject to powerful magnetic field, similar to "micro-accelerators".

Phenomena involving multiple-path analysis should not be forgotten, when considering high or medium energy physics.

In fact, the above discussion suggests the possibility that the neutrino is just a misunderstood consequence of on electron being distributed over a non-simply connected charge distribution, for instance in beta or mesonic decay, and the 1-loop momentum therefore overlooked in the 3-body scattering analysis is the origin of the experimental fact that the energy of the emerging, measured electron, has a continuum spectrum.

Overall, the origin of mass is suggested to be of topological and group of symmetries origin, as it will be analyzed in a subsequent article [3].

The topological aspects demand a holistic approach, i.e. modeling the experiments as networks processing quantum information, rather then the traditional "billiard-ball game" of scattering experiments [19]. The preparation stage (accumulation/transient phase), resonant process and decay, including the strong measurement associated with the output, should be accounted for together, rather then in the "western medicine" vain, by dissection and isolation of individual parts [16].

${ }^{12}$ Things are more complicated computationally, of course; e.g. $S$-matrix and $t \rightarrow \pm \infty$ operators etc. But there should be a "global" reason why physics is "conformal" ...

${ }^{13}$ Corresponding to the decomposition of a graded algebra into its symmetric and antisymmetric part; this would justify why Pauli's exclusion principle holds. 


\section{Conflicts of Interest}

The author declares no conflicts of interest regarding the publication of this paper.

\section{References}

[1] Nambu, Y. (1952) An Empirical Mass Spectrum of Elementary Particles. Progress of Theoretical Physics, 7, 131. https://doi.org/10.1143/ptp/7.2.131

[2] Mac Gregor, M.H. (2007) The Power of $\alpha$ (Alfa). World Scientific, Singapore.

[3] Ionescu, L.M. (2018) On Mass Formulas and Periods, in Preparation.

[4] Castillo, L.R.F. A (Very) Brief History of Particle Physics, PHYS 4470 Nuclear and Particle Physics.

http://www.phy.cuhk.edu.hk/course/2017-2018/2/phys4470/download/0/02.\%20His tory.pdf

[5] Wikipedia, Pion. https://en.wikipedia.org/wiki/Pion

[6] Wiki, Muon. https://en.wikipedia.org/wiki/Muon

[7] Su, Q., Peverly, P.J., Wagner, R.E., Krekora, P. and Grobe, R. Relativistic Electron Spin Motion in Cycloatoms.

https://www.osapublishing.org/oe/abstract.cfm?uri=oe-8-2-51

[8] Wikiquote: Niels Bohr. https://en.wikiquote.org/wiki/Niels Bohr

[9] Peverly, P.J., Wagner, R.E., Su, Q. and Grobe, R. (2000) Fractional Resonances in Relativistic Magnetic-Laser-Atom Interactions. Laser Physics, 10, 303.

[10] Su, Q., Wagner, R.E., Peverly, P.J. and Grobe, R. (2000) Spatial Electron Clouds at Fractional and Multiple Magnetooptical Resonances. In: Xu, Z., Xie, S., Zhu, S.-Y. and Scully, M.O., Eds., Frontiers of Laser Physics and Quantum Optics, Springer, Berlin, 117. https://doi.org/10.1007/978-3-662-07313-1 9

[11] Physics (2000) Focus: Fast Electrons on the Cheap. Physical Review Focus, 5, 15.

[12] Palazzi, P. Particles and Shells. https://arxiv.org/pdf/physics/0301074.pdf

[13] Ionescu, L.M. (2018) Quantizing the Qubit, Work in Progress.

[14] Ionescu, L.M. On the Arrow of Time. https://arxiv.org/abs/0708.4180

[15] Capra, F. The Tao of Physics. https://en.wikipedia.org/wiki/The Tao of Physics

[16] Ionescu, L.M. (2017) A Holistic Approach to Quantum Physics. AASCIT Communications, 4, 19-26.

[17] Kontsevich, M. (1992) Feynman Diagrams and Low-Dimensional Topology, First European Congress of Mathematics Paris. Progress in Mathematics, 120, 97-121. https://doi.org/10.1007/978-3-0348-9112-7 5

[18] Search and See: Slow Light, Optical Molasses, Twisting Light Etc.

[19] Ionescu, L.M. (2018) A Note on Manifolds and Networks as Models in Modern Physics. http://my.ilstu.edu/lmiones/ 